\title{
DÜBLIN
}

Technological University Dublin

ARROW@TU Dublin

\section{Quantification of rubidium as a trace element in beef using laser induced breakdown spectroscopy}

\author{
Yash Dixit \\ Technological University Dublin \\ Maria Piedad Casado-Gavalda \\ Technological University Dublin, maria.casado@tudublin.ie \\ Raquel Cama-Moncunill \\ Technological University Dublin, raquel.cama@tudublin.ie
}

See next page for additional authors

Follow this and additional works at: https://arrow.tudublin.ie/schfsehart

Part of the Chemistry Commons, and the Food Chemistry Commons

\section{Recommended Citation}

Dixit, Yash \& Casado, Maria \& Cama-Moncunill, Raquel \& Cama, Xavier \& Cullen, P.J \& Sullivan, Carl \& Markiewicz-Keszycka, Maria. (2017). Quantification of rubidium as a trace element in beef using laser induced breakdown spectroscopy. Meat Science. -. 10.1016/j.meatsci.2017.03.013.

This Article is brought to you for free and open access by the School of Food Science and Environmental Health at ARROW@TU Dublin. It has been accepted for inclusion in Articles by an authorized administrator of ARROW@TU Dublin. For more information, please contact arrow.admin@tudublin.ie, aisling.coyne@tudublin.ie, gerard.connolly@tudublin.ie.

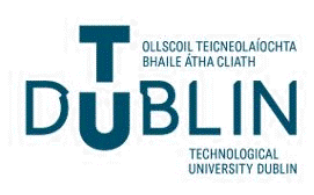




\section{Authors}

Yash Dixit, Maria Piedad Casado-Gavalda, Raquel Cama-Moncunill, Xavier Cama-Moncunill, Patrick J. Cullen, and Carl Sullivan 
1 Quantification of rubidium as a trace element in beef using laser induced breakdown spectroscopy

2 Y. Dixit ${ }^{\mathrm{a}, *}$, Maria P. Casado-Gavalda ${ }^{\mathrm{a}}$, R. Cama-Moncunill ${ }^{\mathrm{a}}$, X. Cama-Moncunill ${ }^{\mathrm{a}}$, P. J. Cullen ${ }^{\mathrm{a}, \mathrm{b}}$, 3 Carl Sullivan ${ }^{\mathrm{a}}$

$4 \quad$ aSchool of Food Science and Environmental Health, Dublin Institute of Technology, Dublin 1, Ireland.

$5 \quad{ }^{b}$ School of Chemical Engineering, University of New South Wales, Sydney, Australia.

$7 *$ Corresponding author:

$8 \quad$ Y. Dixit

9 School of Food Science and Environmental Health, 10

Dublin Institute of Technology, Cathal Brugha St, Dublin 1, Ireland

14 Tel: +35314024543

15 E-mail: yash.dixit@mydit.ie

16

17

18 Keywords: LIBS, minced beef, rubidium, PLSR, chemometrics.

19 
Abstract.

This study evaluates the potential of laser induced breakdown spectroscopy (LIBS) coupled with chemometrics to develop a quantification model for rubidium $(\mathrm{Rb})$ in minced beef. A LIBSCAN 150 system was used to collect LIBS spectra of minced beef samples. Beef liver was used to spike the Rb levels in minced beef. All samples were dried, powdered and pelleted using a hydraulic press.

26 Measurements were conducted by scanning 100 different locations with an automated XYZ sample chamber. Partial least squares regression (PLSR) was used to develop the calibration model, yielding a calibration coefficient of determination $\left(R_{C}^{2}\right)$ of 0.99 and a root mean square error of calibration (RMSEC) of $0.05 \mathrm{ppm}$. The model also showed good results with leave-one-out cross validation, yielding a crossvalidation coefficient of determination $\left(R_{c v}^{2}\right)$ of 0.90 and a root mean square error of cross-validation (RMSECV) of $0.22 \mathrm{ppm}$. The current study shows the potential of LIBS as a rapid analysis tool for the meat processing industry. 


\section{Introduction}

Rubidium $(\mathrm{Rb})$ is a quasi-essential element for living organisms. The human body treats Rb ions similar to potassium ions, concentrating Rb in the body's intracellular fluid (Kékedy-Nagy et al., 2000). A study in 2008 showed that rubidium exhibits anti-depressant properties (Canavese et al., 2008). The daily human intake of $\mathrm{Rb}$ is $\sim 3 \mathrm{mg}$, by foods such as beef, soybeans etc. and drinking water. Beef is a rich source of important trace elements such as iron, copper, zinc as well as rubidium. Beef contains around $27.2 \%$ dry matter out of which 6.84 ppm is rubidium (Fischer, 1997).

Laser induced breakdown spectroscopy (LIBS) is an emerging technique in the field of food analysis. Techniques such as ICP-OES (Inductively coupled plasma - Optical emission spectrometry) and AAS (Atomic absorption spectroscopy) have several limitations such as lengthy sample preparation techniques and the use of hazardous chemicals. LIBS offers various advantages such as minimal sample preparation, chemical free process, rapid detection and portability (Bilge et al., 2016; Kongbonga et al., 2014; Moncayo et al., 2016). Additionally, LIBS also offers the advantage of providing spatial information. Recently, LIBS has been evaluated for mineral quantification in various foods. Singh et al. (2016) used LIBS for quantification of minerals in cucurbit seeds. Andersen et al. (2016) used LIBS to quantify calcium content in comminuted poultry meat for detecting bone particles in mechanically separated meat (MSM). The aim of this study is to evaluate the performance of LIBS coupled with chemometrics to develop a quantification model for rubidium $(\mathrm{Rb})$ in minced beef.

\section{Materials and methods}

\subsection{Sample Preparation}

Fresh striploin beef steaks and beef liver weighing approximately $500 \mathrm{~g}$ each were purchased from a local butchers shop in Dublin, Ireland. The lean meat from the beef steaks was diced into small cubes; the liver was also diced. All samples were minced separately using a laboratory meat blender (8011G, Waring Laboratory Science, Stamford CT, USA), which was carefully cleaned using an antibacterial washing liquid and dried before each use. Finally, samples were placed overnight in a hot air drying oven maintained at $105^{\circ} \mathrm{C}$ using disposable aluminium dishes.

On the next day, dried samples were ground into powder form by using a laboratory meat blender (8011G, Waring Laboratory Science, Stamford CT, USA) followed by sieving using a mechanical siever (VS 1000, Retsch (U.K.) Limited, Hope valley, U.K.) with a 103-mesh screen for 10 minutes at 70 rpm. Beef liver was used to spike the Rb levels in the minced beef. Two independent batches (15 samples per independent batch) with varying percentages of beef liver mixed with lean beef were prepared. Each sample repeated in triplicates comprised of approximately $400 \mathrm{mg}$ of a powdered mixture of lean beef 
containing $0 \%, 10 \%, 20 \%, 40 \%$ and $100 \%$ of liver (w/w). Samples were then pelleted using a hydraulic press (GS01160, Specac Ltd., Orpington, U.K.) by applying a pressure of 10 tonnes for 3 minutes.

\subsection{Graphite furnace atomic absorption spectroscopy analysis}

The rubidium content of lean beef and beef liver were determined using a graphite furnace atomic absorption spectrophotometer (GFAAS) (AA240Z, Agilent Technologies, Santa Clara, USA). Sample preparation was carried out using the standard method of AOAC (FP-3) with slight modifications; approximately $1 \mathrm{~g}$ of powdered sample was transferred into crucibles and pre-ashed on a hot plate with the careful addition of small drops of purified nitric acid (CAS 7697-37-2, Sigma Aldrich, Inc., Arklow, Ireland) to aid digestion. Once samples were completely charred, they were transferred to a muffle furnace maintained at $550{ }^{\circ} \mathrm{C}$ for 5 hours. Ashes were then dissolved into $50 \mathrm{~mL}$ volumetric flasks with $1 \mathrm{M}$ purified nitric acid (CAS 7697-37-2, Sigma Aldrich, Inc.). A further dilution was performed in order to maintain the mineral concentrations within the GFAAS optimum measuring range for $\mathrm{Rb}$. For quantification, calibration curves were obtained using standard solutions of rubidium (55727, Sigma Aldrich, Inc., Arklow, Ireland). All samples were measured in triplicate.

\subsection{LIBS spectra acquisition}

LIBS spectra were recorded using a LIBSCAN 150 system (Applied Photonics Limited, Skipton North Yorkshire, U.K.) which consists of a Q-switched Nd:YAG laser (ultra, Quantel laser, 601 Haggerty Lane Bozeman, MT, USA), a series of six spectrophotometers covering the wavelength range of 185-904 nm and a LIBSCAN 150 head. The head incorporates a miniature CCD camera and 6 lens holders which collect plasma light of different wavelength regions. The laser used for sample ablation had a pulse energy of $150 \mathrm{~mJ}$ and a pulse duration of $5 \mathrm{~ns}$ operating at $1064 \mathrm{~nm}$. A repetition rate of $1 \mathrm{~Hz}$ was employed along with a $1.27 \mu$ s gate delay and $1.1 \mathrm{~ms}$ integration time in Q-switched mode. The sample was placed at a LTSD (lens to sample distance) of approximately $80 \mathrm{~mm}$ to ensure that the laser was focussed onto the sample. Samples were measured by scanning 100 different locations in a 10 X 10 grid pattern while the sample was moved after each shot with an automated XYZ sample chamber (XYZ-750, Applied Photonics Limited, Skipton North Yorkshire, U.K.) by a step size of $0.70 \mathrm{~mm}$.

\subsection{Data analysis}

Data analysis was performed using R (R Core Team, 2014). The "pls" package (Mevik et al., 2013) was used for performing PLSR (partial least square regression).

Spectral pre-processing is the most important step before performing chemometric bi-linear modelling such as PLSR in order to remove baseline shifts and non-linearities from the acquired spectra (Rinnan et al., 2009). Pre-processing was performed using standard normal variate (SNV) transformation. 
Processed data in the wavelength range of 774.527 to 784.824 was selected because the Rb peak exists in

101 this region at $780.010 \mathrm{~nm}$. In order to develop the calibration model, the acquired processed data along

102 with the $\mathrm{Rb}$ values obtained were subjected to PLSR. The method of leave-one-out was used for cross

103 validation while developing the calibration models in order to avoid either over- or under-fitting of the 104 models. Goodness of fit was evaluated by determining both root mean square error of calibration 105 (RMSEC) and root mean square error in cross validation (RMSECV) which provides information about 106 the deviation of models from their reference values (Cama-Moncunill et al., 2016). The corresponding 107 values of both coefficients of determination in calibration $\left(R_{c}^{2}\right)$ and in cross validation $\left(R_{c v}^{2}\right)$ were also 108 calculated.

\section{Results and Discussion}

\subsubsection{Graphite furnace atomic absorption spectroscopy analysis}

111 GFAAS was performed to determine the concentration of rubidium in pure lean beef and pure liver 112 samples. Results of GFAAS analysis are illustrated in Table 1. GFAAS results indicate that Rb content in 113 lean beef is generally lower than in beef liver. The results shown in Table 1 were in good agreement with 114 those reported in the literature (Fischer, 1997).

\subsubsection{Spectral analysis}

116 Fig. 1a shows the raw LIBS spectra of powdered lean beef and beef liver. Each spectrum corresponds to 117 an average of 100 spectra collected at different locations of the pellet in order to overcome sample 118 heterogeneity. Emission peak related to rubidium in Fig. 1a have been identified at $780.010 \mathrm{~nm}$ with 119 reference to the NIST database (Kramida et al., 2015). Rb exists under group 1 of the periodic table, 120 having a single electron in its outer shell and allowing LIBS to easily excite the lone electron and detect 121 the element (Scerri, 2007). It is evident from Fig. 1b that the LIBS spectra clearly differentiates samples

Best model was identified with four PLS components. The model showed a good fit yielding a $R_{c}^{2}$

\subsubsection{Multivariate data analysis}

In the current study, PLSR was performed on pre-processed LIBS data in order to develop a calibration model for Rb. PLSR generates linear prediction models by optimising the covariance between spectral data and the reference values ( $\mathrm{Rb}$ content). In order to do so, it performs decomposition on both the spectral and reference data simultaneously (Dixit et al., 2016; ElMasry et al., 2013). of 0.99 and $R_{c v}^{2}$ of 0.90 . Moreover, the RMSEC and RMSECV were low yielding values of $0.05 \mathrm{ppm}$ and 
$1310.22 \mathrm{ppm}$ respectively. It could be possible to improve model performance in future studies by optimising 132 factors such as laser pulse energy and LTSD (Radziemski et al., 2006).

133

134

135

136

137

138

139

140

141

142

143

144

\section{Conclusions}

The present study shows that LIBS can be successfully used to detect and quantify the trace element Rb in minced beef and beef liver. PLSR was used to model the LIBS spectral data along with reference values obtained for $\mathrm{Rb}$ using GFAAS. High values of $R_{c v}^{2}$ and low values of the corresponding RMSECV confirmed a good fit for the model. LIBS is an emerging technique in the area of food analysis with potential as an at-line monitoring tool for the meat industry. Results from the current study illustrates high potential for LIBS as a rapid technique to detect and quantify Rb as well as other important trace elements such as iron, zinc and copper amongst others in various foods. However, factors related to sample surface such as surface homogeneity, chemical composition and particle size can affect the LIBS spectra. Effects due to non-homogeneous sample surface and chemical composition could be reduced by sufficient averaging so that a better representation of the sample could be obtained. Furthermore, variation in particle size produces a matrix effect which could be reduced with matrix-matched calibration standards.

In order to make LIBS a suitable technique for routine analysis in a food industry further improvements are required such as an automated focusing system for optimized LTSD and use of detectors optimized for the spectral region of interest. Overall, the study demonstrates potential for LIBS as a rapid analysis tool for the meat processing industry.

\section{Acknowledgements}

The authors would like to acknowledge funding from the Food Institutional Research Measure administered by the Department of Agriculture, Food and the Marine, Ireland (Grant agreement: $13 / \mathrm{F} / 508)$.

\section{References}

Andersen, M.-B. S., Frydenvang, J., Henckel, P., \& Rinnan, Å. (2016). The potential of laser-induced breakdown spectroscopy for industrial at-line monitoring of calcium content in comminuted poultry meat. Food Control, 64, 226-233. doi: 10.1016/j.foodcont.2016.01.001

Bilge, G., Velioglu, H. M., Sezer, B., Eseller, K. E., \& Boyaci, I. H. (2016). Identification of meat species by using laser-induced breakdown spectroscopy. Meat Science, 119, 118-122. doi: 10.1016/j.meatsci.2016.04.035 
160

161

162

163 164

165

166

167

168

169

170

171

172

173

174

175

176

177

178

179

180

181

182

183

184

185

186

187

Cama-Moncunill, R., Markiewicz-Keszycka, M., Dixit, Y., Cama-Moncunill, X., Casado-Gavalda, M. P., Cullen, P. J., et al. (2016). Multipoint NIR spectroscopy for gross composition analysis of powdered infant formula under various motion conditions. Talanta, 154, 423-430.

Canavese, C., Decostanzi, E., Bergamo, D., Sabbioni, E., \& Stratta, P. (2008). Rubidium, salami and depression. You cannot have everything in life. Blood Purification, 26(4), 311-314. doi: $10.1159 / 000129657$

Dixit, Y., Casado-Gavalda, M. P., Cama-Moncunill, R., Cama-Moncunill, X., Jacoby, F., Cullen, P. J., et al. (2016). Multipoint NIR spectrometry and collimated light for predicting the composition of meat samples with high standoff distances. Journal of Food Engineering, 175, 58-64.

ElMasry, G., Sun, D.-W., \& Allen, P. (2013). Chemical-free assessment and mapping of major constituents in beef using hyperspectral imaging. Journal of Food Engineering, 117(2), 235-246.

Fischer, P. W. F. (1997). Trace Elements in Man and Animals--9: Proceedings of the Ninth International Symposium on Trace Elements on Man and Animals. Ottawa, Canada: Canadian Science Publishing, NRC Research Press.

Kékedy-Nagy, L., \& Cordoş, E. A. (2000). Flame atomic emission determination of rubidium in mineral and well waters using methane-air flame as excitation source. Talanta, 52(4), 645-652.

Kongbonga, Y. G. M., Ghalila, H., Onana, M. B., \& Lakhdar, Z. B. (2014). Classification of vegetable oils based on their concentration of saturated fatty acids using laser induced breakdown spectroscopy (LIBS). Food Chemistry, 147, 327-331.

Kramida, A., Ralchenko, Y., Reader, J., \& Team, N. A. (2015). NIST Atomic Spectra Database (Ver. 5.3), [Online]. Retrieved 2016, October 6 http://physics.nist.gov/asd

Mevik, B.-H., Wehrens, R., \& Liland, K. H. (2013). pls: Partial least squares and principal component regression. $R$ package version, 2.4-3.

Moncayo, S., Rosales, J. D., Izquierdo-Hornillos, R., Anzano, J., \& Caceres, J. O. (2016). Classification of red wine based on its protected designation of origin (PDO) using Laser-induced Breakdown Spectroscopy (LIBS). Talanta, 158, 185-191. doi: 10.1016/j.talanta.2016.05.059

R Core Team. (2014). R: A language and environment for statistical computing. R Foundation for Statistical Computing, Vienna, Austria, 2012: ISBN 3-900051-07-0. 
188 Radziemski, L. J., \& Cremers, D. A. (2006). Handbook of Laser Induced Breakdown Spectroscopy. West 189 Sussex, England: John Wiley \& Sons.

190 Rinnan, Å, van den Berg, F., \& Engelsen, S. B. (2009). Review of the most common pre-processing 191 techniques for near-infrared spectra. TrAC Trends in Analytical Chemistry, 28(10), 1201-1222.

192 Scerri, E. R. (2007). The periodic table: its story and its significance. NY, USA: OUP

193 Singh, J., Kumar, R., Awasthi, S., Singh, V., \& Rai, A. (2016). Laser Induced breakdown spectroscopy: 194 A rapid tool for the identification and quantification of minerals in cucurbit seeds. Food $195 \quad$ Chemistry.

196 7. Figure captions

197 Fig. 1 LIBS spectra: (a) raw spectra of lean beef and beef liver, (b) SNV transformed spectra: from 198 top to bottom - rubidium peak at $780 \mathbf{~ n m}$ for batches 1 and 2 . The arrow ( $\uparrow$ ) indicates the direction 199 of increasing content of rubidium. 